\title{
Sarcoidosis with Pancreatic Mass, Endobronchial Nodules, and Miliary Opacities in the Lung
}

\author{
Shun Matsuura ${ }^{1}$, Yasutaka Mochizuka ${ }^{1}$, Kyohei Oishi $^{1}$, Koichi Miyashita ${ }^{1}$, Hyogo Naoi $^{1}$, \\ Eisuke Mochizuki ${ }^{1}$, Shinichiro Mikura ${ }^{1}$, Masaru Tsukui ${ }^{1}$, Naoki Koshimizu ${ }^{1}$, \\ Akihiko Ohata $^{2}$ and Takahumi Suda ${ }^{3}$
}

\begin{abstract}
:
Sarcoidosis affects multiple organs and rarely has unusual manifestations. A 78-year-old woman was referred to our hospital for coughing symptoms. A chest computed tomography (CT) scan revealed bilateral diffuse miliary patterns and right pleural effusion. Bronchoscopy showed multiple nodules in the carina and the bronchus intermedius. A CT scan of her abdomen revealed hypovascular lesions involving the pancreatic head and body. A transbronchial lung biopsy, bronchial mucosal biopsy, and endoscopic ultrasound-guided fine-needle aspiration of the pancreatic mass demonstrated non-caseating granulomas. We diagnosed the patient with sarcoidosis. She received no treatment for sarcoidosis and has been followed up for one year, during which no pulmonary disease progression had been observed and the pancreatic masses partially regressed.
\end{abstract}

Key words: pleural effusion, pancreatic sarcoidosis, miliary opacities, endobronchial nodules, sarcoidosis

(Intern Med 56: 3083-3087, 2017)

(DOI: 10.2169/internalmedicine.8916-17)

\section{Introduction}

Sarcoidosis is a chronic granulomatous disease of unknown etiology. Because sarcoidosis can involve any organ system, the clinical presentation is often variable. Pleural effusion, endobronchial nodules, and miliary opacities in the lung are its unusual manifestations, but the simultaneous presence of these three rare events in this disease has not been reported.

Pancreatic sarcoidosis is particularly rare. The clinical and radiological findings of pancreatic sarcoidosis overlap with those of malignancy, making a diagnosis difficult. Most reports have described cases of pancreatic sarcoidosis diagnosed using a surgical biopsy $(1,2)$; however, we diagnosed a case of pancreatic sarcoidosis using endoscopic ultrasound-guided fine-needle aspiration (EUS-FNA).

We herein report a rare case of pancreatic sarcoidosis with endobronchial abnormality and miliary opacities.

\section{Case Report}

A 78-year-old woman presented to our hospital with a 2month history of coughing. She had no abdominal pain, nausea, vomiting, or weight loss. She denied alcohol and tobacco use. Her medical history included dementia. Her vital signs were within normal limits. She had no lymphadenopathy of the head and neck or subcutaneous nodules in her skin. Her serum amylase level was slightly elevated (Table). Chest radiography revealed miliary nodules and pleural effusion (Fig. 1A). A chest computed tomography (CT) scan revealed bilateral diffuse micronodular shadow, thickening of the bronchovascular bundles, curvilinear shadow, and right pleural effusion (Fig. 1B-D). There was no evidence of bilateral hilar lymphadenopathy. A differential diagnosis of miliary tuberculosis, metastatic pulmonary carcinoma, and sarcoidosis was considered. Diagnostic thoracentesis with a pleural fluid analysis was performed in which pleural effusion was revealed to be a lymphocyte-predominant exudate.

${ }^{1}$ Division of Respiratory Internal Medicine, Fujieda Municipal General Hospital, Japan, ${ }^{2}$ Division of Gastroenterology, Fujieda Municipal General Hospital, Japan and ${ }^{3}$ Second Division, Department of Internal Medicine, Hamamatsu University School of Medicine, Japan 
Table. Laboratory Findings.

\begin{tabular}{lclrlr}
\hline \multicolumn{2}{c}{$<$ Blood Cell Counts $>$} & \multicolumn{2}{c}{$<$ Blood Chemistry } & \multicolumn{2}{c}{$<$ Serological Study $>$} \\
\hline WBC & $5,700 / \mu \mathrm{L}$ & T-bil & $0.3 \mathrm{mg} / \mathrm{dL}$ & CEA & $3.4 \mathrm{ng} / \mathrm{mL}$ \\
Neut & $69.7 \%$ & AST & $17 \mathrm{IU} / \mathrm{L}$ & CYFRA & $1.7 \mathrm{ng} / \mathrm{mL}$ \\
Lymph & $2.6 \%$ & ALT & $8 \mathrm{IU} / \mathrm{L}$ & ProGRP & $32.7 \mathrm{pg} / \mathrm{mL}$ \\
Eosino & $0.4 \%$ & LDH & $189 \mathrm{IU} / \mathrm{L}$ & IL2 receptor & $1,570 \mathrm{U} / \mathrm{mL}$ \\
Baso & $21.0 \%$ & ALP & $181 \mathrm{IU} / \mathrm{L}$ & & \\
Mono & $6.3 \%$ & $\gamma-\mathrm{GTP}$ & $24 \mathrm{IU} / \mathrm{L}$ & T-SPOT & $(-)$ \\
RBC & $371 \times 10^{4} / \mu \mathrm{L}$ & AMY & $335 \mathrm{IU} / \mathrm{L}$ & MAC antibody & $(-)$ \\
Hb & $10.8 \mathrm{~g} / \mathrm{dL}$ & $\mathrm{BUN}$ & $18 \mathrm{mg} / \mathrm{dL}$ & & \\
Hct & $33.9 \%$ & $\mathrm{Cre}$ & $0.7 \mathrm{mg} / \mathrm{dL}$ & IgG & $1,492 \mathrm{mg} / \mathrm{dL}$ \\
PLT & $20.7 \times 10^{4} / \mu \mathrm{L}$ & $\mathrm{Na}$ & $143 \mathrm{mEq} / \mathrm{L}$ & IgG4 & $65.7 \mathrm{mg} / \mathrm{dL}$ \\
& & $\mathrm{K}$ & $3.9 \mathrm{mEq} / \mathrm{L}$ & & \\
& & $\mathrm{Cl}$ & $108 \mathrm{mEq} / \mathrm{L}$ & ACE & $51.7 \mathrm{U} / \mathrm{L}$ \\
& $\mathrm{Ca}$ & $9.1 \mathrm{mg} / \mathrm{dL}$ & & \\
& & $\mathrm{P}$ & $4.0 \mathrm{mg} / \mathrm{dL}$ & & \\
\hline
\end{tabular}
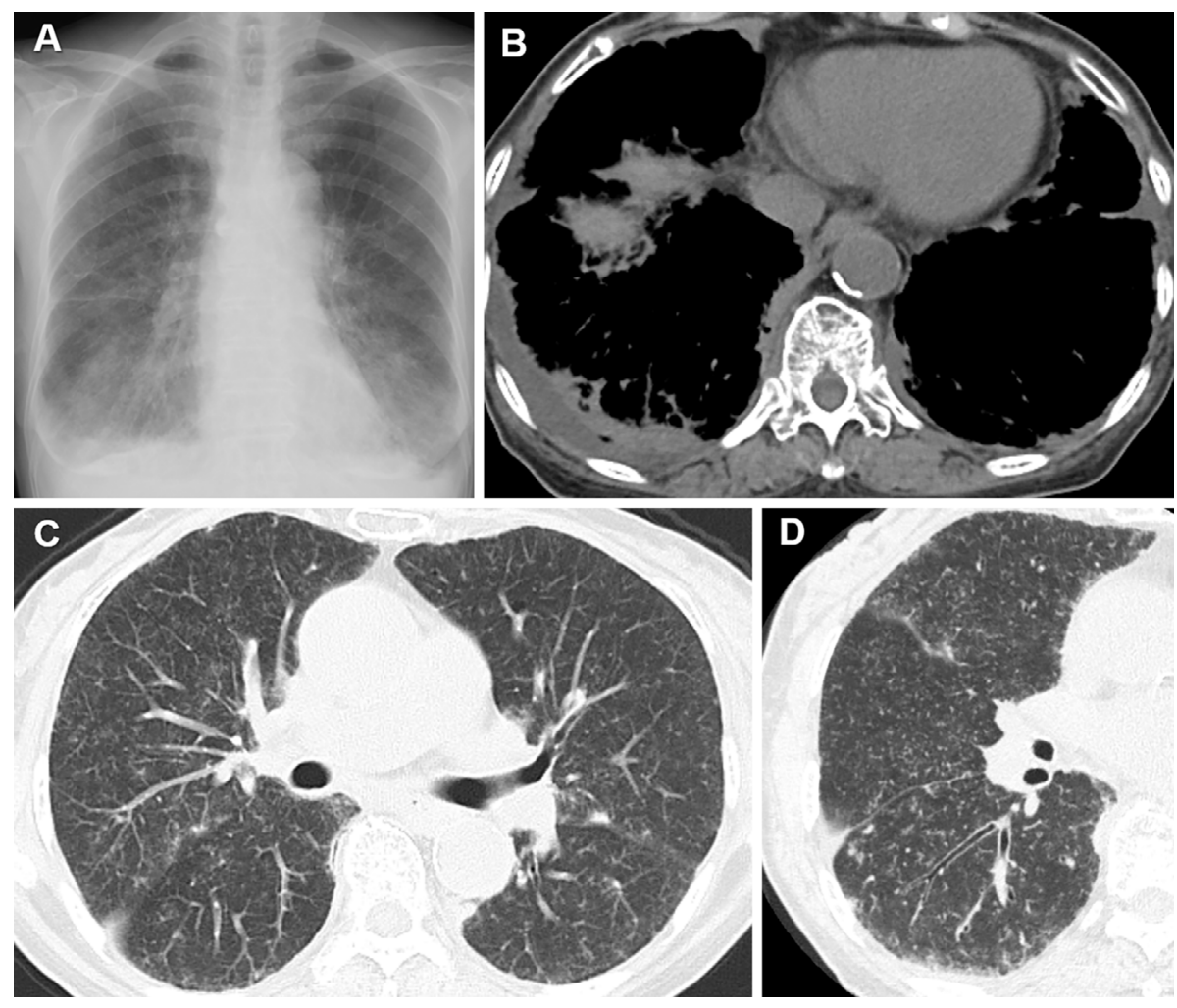

Figure 1. A) Pleural effusion and miliary opacities in sarcoidosis. B) A computed tomography (CT) scan shows right pleural effusion with compression of the right lower lung. C) Numerous tiny micronodules representing a random distribution. D) High-resolution CT: Perilymphatic nodules located in fissures and subpleural regions, with bronchial wall thickening.

Mycobacterial culture and cytological findings were negative. Bronchoscopy with bronchoalveolar lavage (BAL) and a transbronchial lung biopsy (TBLB) were performed. An evaluation of the endobronchial characteristics revealed multiple nodules (Fig. 2). The BAL fluid of the right middle lobe revealed lymphocytosis $(64.0 \%)$ and an elevated CD4/8 ratio of 8.69. A bronchial mucosal biopsy and TBLB demonstrated non-caseating granulomas (Fig. 3A and B). We diagnosed the patient with sarcoidosis, and a subsequent sys- temic search was performed.

The serum angiotensin-converting enzyme level was increased at 51.7 U/L (Table). Cardiovascular exams were unremarkable. Lung function testing revealed a vital capacity (VC) of $2.20 \mathrm{~L}(105.8 \%)$, forced expiratory volume in 1 second (FEV1) of $1.98 \mathrm{~L}$ (123.7\%), FEV1/forced vital capacity (FVC) of $85.7 \%$, maximum flow at $50 \%$ vital capacity (V50) of $1.97 \mathrm{~L} / \mathrm{s}(60.2 \%)$, maximum flow at $25 \%$ vital capacity (V25) of $0.91 \mathrm{~L} / \mathrm{s}(103.4 \%)$, V50/V25 ratio of 2.16, 


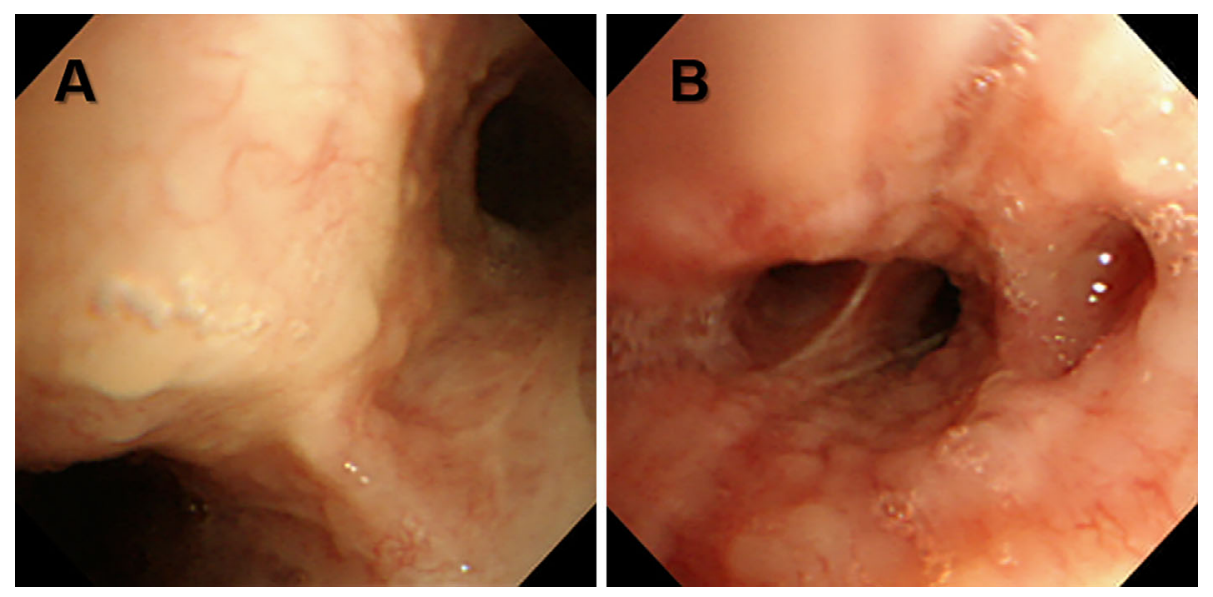

Figure 2. Bronchoscopy shows multiple variable small endobronchial nodules $\mathbf{A}$ ) in the carina and B) in the bronchus intermedius.
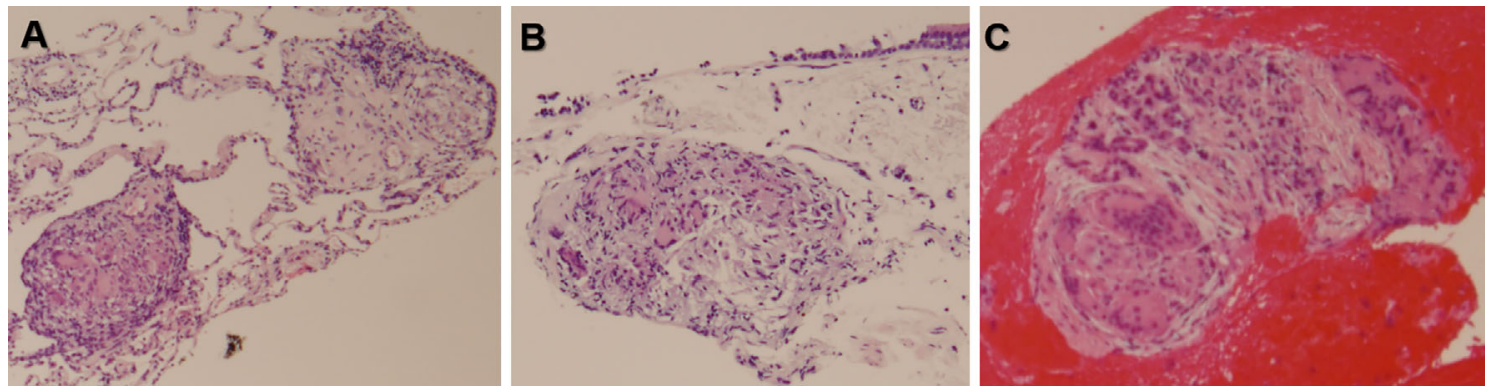

Figure 3. Noncaseating granulomas. A) Transbronchial lung biopsy. B) Bronchial mucosal biopsy.

C) Endoscopic ultrasound-guided fine-needle aspiration of the pancreatic mass.

and diffusing capacity of the lung for carbon monoxide (DLCO) of $7.86 \mathrm{~mL} / \mathrm{min} / \mathrm{mmHg}(62.0 \%)$. No uveitis was identified on an ophthalmic examination. A CT scan of her abdomen revealed hypovascular lesions involving the pancreatic head and body and the dilation of pancreatic duct (Fig. 4A and B). Magnetic resonance imaging revealed a well-defined mass with high signal intensity on the diffusion-weighted images of the same region (Fig. 4C and D) and pancreatic duct disruption (Fig. 4E). EUS-FNA was performed, revealing a hypoechoic mass with a clear outline in the pancreatic head and body (Fig. 4F). The lesion cytology was negative for neoplastic cells. Pathological findings revealed non-caseating epithelioid granulomas (Fig. 3C). The ultimate diagnosis was pancreatic, pulmonary, and bronchial sarcoidosis.

Her cough spontaneously improved. She received no treatment for sarcoidosis and was followed up at an out-patient clinic for one year, during which no pulmonary disease progression was observed and the pancreatic masses partially regressed (Fig. 5).

\section{Discussion}

Although sarcoidosis involving thoracic lymph nodes and pulmonary parenchyma is familiar to most clinicians, pulmonary sarcoidosis infiltrating the bronchi is rare. In previ- ous reports, the results of endobronchial biopsy correlated with abnormal airway appearance, but the endobronchial status with sarcoidosis was not associated with airflow obstruction (3). In previous studies reporting different results, $14-63 \%$ of patients with sarcoidosis had obstructive airway dysfunction (4-6). Small airway dysfunction without a restrictive defect is common in sarcoidosis cases (7). In this patient, although the spirometry values of VC, FEV1, and FEV1.0/VC ratio were within normal limits, the DLCO and V50 were decreased. As observed in previous reports, although endobronchial disease may not play a role in the development of airflow obstruction in sarcoidosis, parenchymal involvement is associated with small airway dysfunction and a decreased diffusion capacity.

Miliary patterns are rare in sarcoidosis $(<1 \%$ of cases) (8). The most common conditions with miliary patterns are miliary tuberculosis, pneumoconiosis, and metastatic lesions. The predominance and distribution of nodules in relation to the secondary lung lobules may be helpful for a differential diagnosis (9). Three types of distributions have been described: centrilobular, perilymphatic, and random. In our patient, nodular thickening of the interlobular septa and areas of ground-glass opacity in the lungs were revealed on high-resolution CT. These imaging findings suggested a perilymphatic distribution, rather than a random one. Miliary opacities of sarcoidosis are the most common processes that 

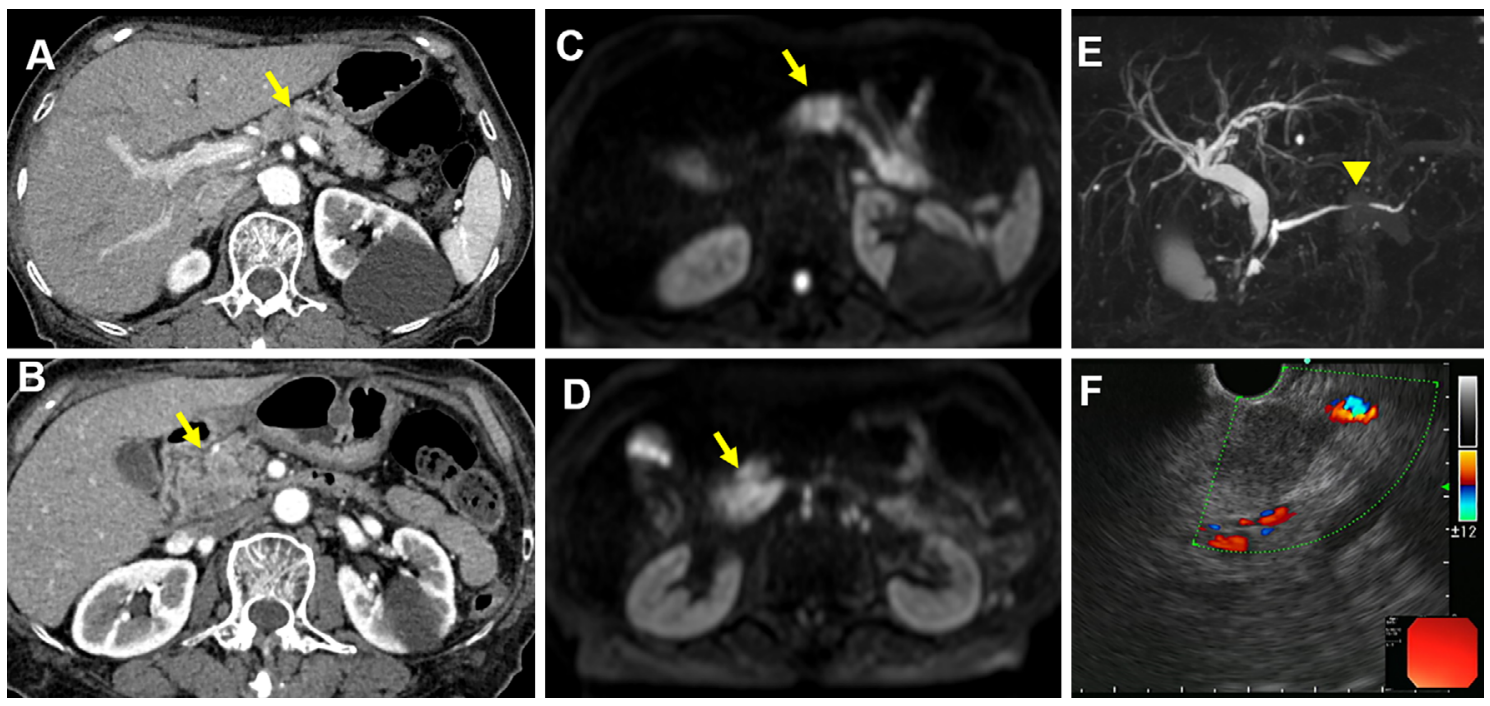

Figure 4. Pancreatic sarcoidosis. A) and B) A dynamic enhanced computed tomography scan shows the mass in the pancreatic head (arrow) and body (arrow). C) and D) Magnetic resonance cholangiopancreatography reveals a mass in the pancreatic head and body. E) Disruption of the main pancreatic duct (arrow head). F) Endoscopic ultrasound via the stomach reveals a hypoechoic mass, and fine-needle aspiration is performed.
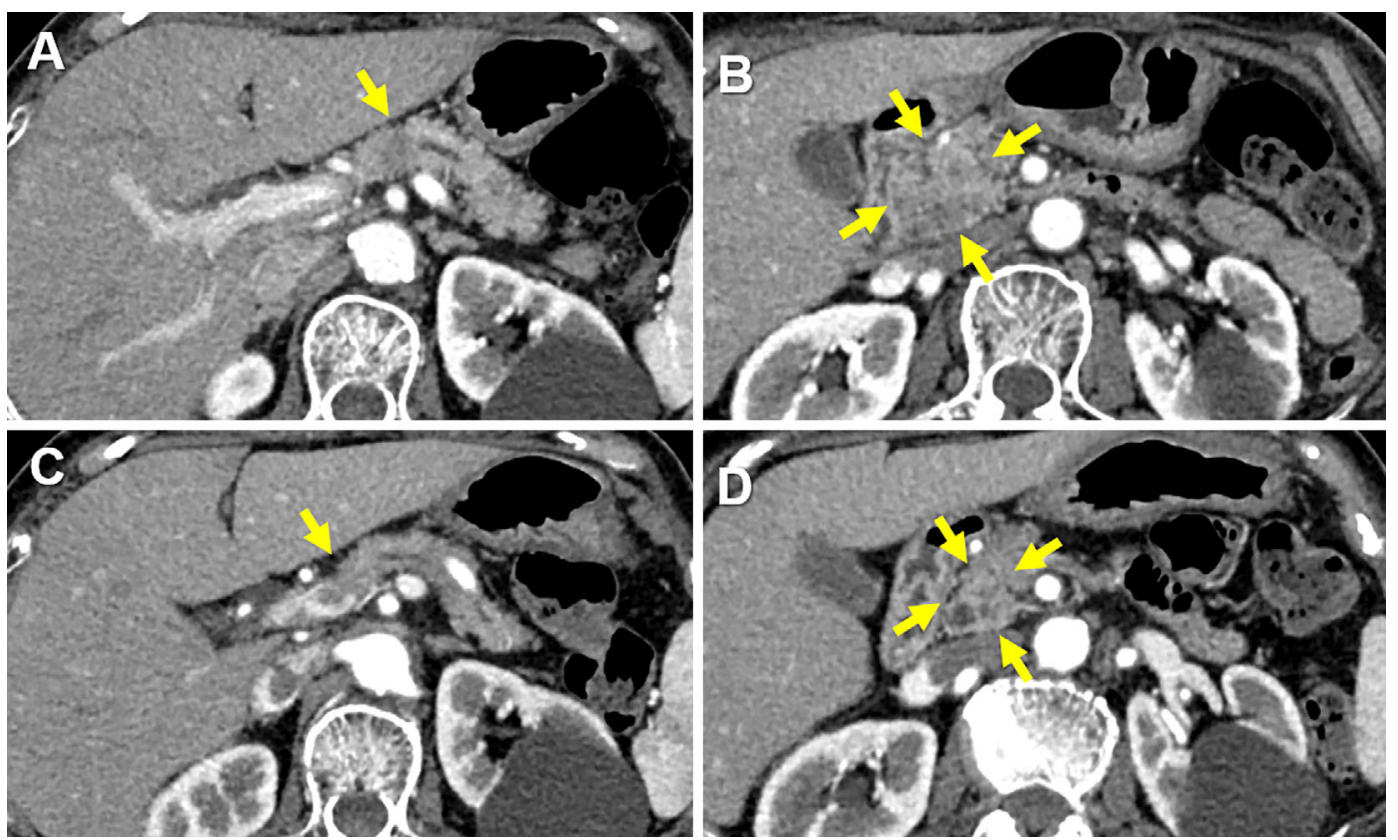

Figure 5. A) and B) A dynamic enhanced computed tomography scan reveals masses in the pancreatic head (arrows) and body (arrow), measuring $25.0 \times 33.1 \mathrm{~mm}$ and $12.7 \times 11.0 \mathrm{~mm}$, respectively. C and D) The masses shrank to $21.8 \times 19.3 \mathrm{~mm}$ and $10.0 \times 9.0 \mathrm{~mm}$, respectively, at one-year follow-up without treatment.

exhibit perilymphatic distribution.

In a previous series of 181 patients with sarcoidosis, the prevalence of all pleural effusion was $2.8 \%$, with pleural effusion caused by sarcoidosis found in only $1.1 \%$ of patients (10). We did not perform thoracoscopy in the present patient; therefore, we were unable to make a definitive diagnosis of sarcoidosis pleural effusion. Because of the absence of malignancies and acid-fast bacilli in the pleural effusion, we thought that the effusion might be associated with pulmonary infiltrate or pleural involvement in sarcoidosis. Thoracentesis or a pleural biopsy is necessary to rule out malignancy or infection that can mimic sarcoidosis pleural effusion.

Pancreatic sarcoidosis is only very rarely detected before death. At autopsy, only $1-5 \%$ of the patients with systemic or disseminated sarcoidosis have sarcoid lesions within the 
pancreas $(11,12)$. In 2006, Caceres et al. reported 25 patients with surgically proven pancreatic sarcoidosis. Symptomatic pancreatic sarcoidosis most commonly presented with abdominal pain (15 patients), weight loss (11 patients), obstructive jaundice ( 7 patients), and emesis (5 patients), conversely, no abdominal symptom was observed in four patients (1). Our patients had no abdominal symptoms, and the pancreatic mass was incidentally found during a systemic search. The differentiation of pancreatic sarcoidosis from pancreatic cancer is difficult, and a surgical biopsy is needed in most cases (13-16). When a benign pancreatic mass is identified before conducting a surgical biopsy for pancreatic sarcoidosis, a method of judging the response to steroid treatment can be applied (17). In asymptomatic patients with sarcoidosis, the side effects of systemic steroids often exceed the treatment benefits, and the disease may spontaneously remit (18). The patient in the present case was asymptomatic and did not undergo steroid therapy; however, the pancreatic mass still partially shrank in size.

Our patient was diagnosed using EUS-FNA and not surgery. EUS-FNA is a safe, minimally invasive, accurate outpatient procedure for the cytological diagnosis of many benign and malignant conditions (19). A cytological examination using EUS-FNA to obtain a specimen of the pancreatic mass was found to have higher sensitivity and accuracy than endoscopic retrograde cholangiopancreatography (20). To our knowledge, this is the first reported case of the successful diagnosis of pancreatic sarcoidosis by EUS-FNA, which may prevent unnecessary operations in patients with pancreatic masses associated with sarcoidosis.

In conclusion, we experienced a case of sarcoidosis with pancreatic, pulmonary, and bronchial lesions. Our initial findings from the radiographic image of pleural effusion and miliary opacities indicated miliary tuberculosis or a metastatic pulmonary tumor; however, our patient was diagnosed with sarcoidosis based on the endobronchial nodular involvement and pathology using a TBLB. We diagnosed the pancreatic mass, which was detected by a whole-body evaluation, as pancreatic sarcoidosis using noninvasive EUS-FNA.

The authors state that they have no Conflict of Interest (COI).

\section{Acknowledgement}

I would like to thank Ms. H. Oshio (Tokyo University) for her useful advice.

\section{References}

1. Caceres M, Sabbaghian MS, Braud R, Wilks S, Boyle M. Pancreatic sarcoidosis: unusual presentation resembling a periampullary malignancy. Curr Surg 63: 179-185, 2006.

2. Al-Sukhni E, Qiu J, Gabriel E, Hochwald S. Sarcoidosis present- ing with primary pancreatic manifestations: a case report and review of the literature. J Mol Biomark Diagn 7: 290, 2016.

3. Shorr AF, Torrington KG, Hnatiuk OW. Endobronchial involvement and airway hyperreactivity in patients with sarcoidosis. Chest 120: 881-886, 2001.

4. Sharma OP, Johnson R. Airway obstruction in sarcoidosis. A study of 123 nonsmoking black American patients with sarcoidosis. Chest 94: 343-346, 1988.

5. Gibson GJ, Prescott RJ, Muers MF, et al. British Thoracic Society sarcoidosis study: effect of long-term corticosteroid treatment. Thorax 51: 238-247, 1996.

6. Baughman RP, Teirstein AS, Judson MA, et al. Clinical characteristics of patients in a case control study of sarcoidosis. Am J Respir Crit Care Med 164: 1885-1889, 2001.

7. Argyropoulou PK, Patakas DA, Louridas GE. Airway function in stage I and stage II pulmonary sarcoidosis. Respiration 46: 17-25, 1984.

8. Criado E, Sánchez M, Ramirez J, et al. Pulmonary sarcoidosis: typical and atypical manifestations at high-resolution $\mathrm{CT}$ with pathologic correlation. Radiographics 30: 1567-1586, 2010.

9. Andreu J, Mauleón S, Pallisa E, Majó J, Martinez-Rodriguez M, Cáceres J. Miliary lung disease revisited. Curr Probl Diagn Radiol 31: 189-197, 2002.

10. Huggins JT, Doelken P, Sahn SA, King L, Judson MA. Pleural effusions in a series of 181 outpatients with sarcoidosis. Chest 129: 1599-1604, 2006.

11. Harder H, Buchler MW, Frohlich B, et al. Extrapulmonary sarcoidosis of liver and pancreas: a case report and review of literature. World J Gastroenterol 13: 2504-2509, 2007.

12. Iwai K, Tachibana T, Hosoda Y, Matsui Y. Sarcoidosis autopsies in Japan. Frequency and trend in the last 28 years. Sarcoidosis 5: 60$65,1988$.

13. Siavelis HA, Herrmann ME, Aranha GV, Garcia G, Eubanks T, Reyes CV. Sarcoidosis and the pancreas. Surgery 125: 456-461, 1999.

14. Bacal D, Hoshal VL, Schaldenbrand JD, Lampman RM. Sarcoidosis of the pancreas: case report and review of the literature. Am Surg 66: 675-678, 2000.

15. Ohana G, Melki Y, Rosenblat Y, Kravarusic D, Weil R. Pancreatic sarcoidosis mimicking a malignant tumour. Eur J Surg 168: 513$515,2002$.

16. Alizadeh L, Alizadeh AHM. Pancreatic sarcoidosis: a literature review. J Pancreas 17: 566-573, 2016.

17. Wolfson D, Barkin J, Chari S, et al. Management of pancreatic masses. Pancreas 31: 203-217, 2005.

18. Mueller S, Boehme MW, Hofmann WJ, Stremmel W. Extrapulmonary sarcoidosis primarily diagnosed in the liver. Scand J Gastroenterol 35: 1003-1008, 2000.

19. Charles ED, Waxman I. Endoscopic ultrasound. Gastroenterol Clin North Am 31: 863-879, 2002.

20. Wakatsuki T, Irisawa A, Bhutani MS, et al. Comparative study of diagnostic value of cytologic sampling by endoscopic ultrasonography-guided fine-needle aspiration and that by endoscopic retrograde pancreatography for the management of pancreatic mass without biliary stricture. J Gastroenterol Hepatol 20: 1707-1711, 2005.

The Internal Medicine is an Open Access article distributed under the Creative Commons Attribution-NonCommercial-NoDerivatives 4.0 International License. To view the details of this license, please visit (https://creativecommons.org/licenses/ by-nc-nd/4.0/).

(C) 2017 The Japanese Society of Internal Medicine Intern Med 56: 3083-3087, 2017 